\title{
Energy Efficient and Improved Network Lifetime Multipath Routing using FF-AOMDV and Dragonfly Topology
}

\author{
Shanti Jaiswal \\ M.Tech Scholar \\ Department of ECE \\ SIRT (Bhopal)
}

\author{
Navneet Kaur \\ Associate Professor \\ Department of ECE \\ SIRT (Bhopal)
}

\begin{abstract}
In Mobile Ad-hoc networks (MANETs), due to restricted power energy offering and frequent topology changes caused by node mobility, routing becomes a difficult problem. The research performed till date highlights this very specific drawback of energy consumption in MANETs and by applying the protocol named Ad-hoc on Demand Multipath Distance Vector with the Fitness function (FF-AOMDV) and Dragonfly topology we had minimized it. The fitness function is used to find the best path from the supply to the destination to reduce the energy consumption in multipath routing by using Dragonfly topology. The performance of the proposed FF-AOMDV protocol with dragonfly topology was evaluated using Network simulator Version 2 (NS-2), where the performance was compared with AOMDV and Ad-hoc on Demand Multipath Routing with Life Maximization (AOMRLM) protocols, the two most popular protocols of this area. The comparison was done to evaluate performance metrics such as energy consumption, throughput, packet delivery ratio, end-to-end delay, network lifetime and routing overhead ratio by varying the node speed, packet size and simulation time. The results clearly demonstrate that the proposed FFAOMDV protocol with Dragonfly topology outperformed AOMDV and AOMR-LM under majority of the network performance metrics and parameters.
\end{abstract}

\section{Keywords}

Energy efficient protocol, Mobile Ad-hoc network, multipath routing, fitness function, and Dragonfly topology

\section{INTRODUCTION}

The term MANET (Mobile ad hoc Network) refers to a multihop packet based wireless network composed of a collection of mobile nodes which will communicate and move at an equivalent time, while not using any kind of mounted wired and fixed infrastructure. Mobile Ad-hoc Networks (MANETs) are assortment of self routing enabled devices that communicate among themselves with none specific network infrastructure. Obviously, these networks are decentralized and believe neighbors for communication [1]. The topology of the networks isn't fixed and is subjected to alter over time because of the mobile nature of the devices.

The network communication between a supply and destination is mostly in multi hop that energy of the devices plays an important role besides quality. MANETs are actually self organizing and adaptive networks that can be formed and deformed on-the-fly while no necessity of any centralized administration. A MANET could be a sort of ad-hoc network which will change locations and configure itself on the fly. Since MANETs are mobile, they use wireless connections to connect to numerous networks. This could be a typical Wi-Fi connection, or another medium, like a cellular or satellite transmission [6].

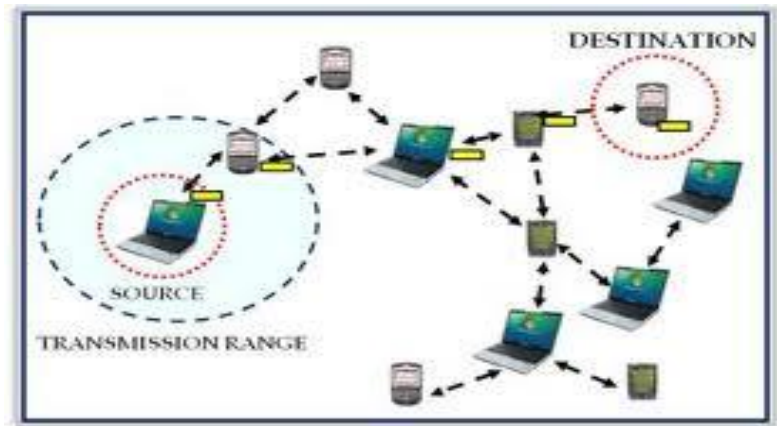

Fig.1 Structure of MANET

The method of routing in energy dependant networks has to meet stability and quality throughout the communication time. Simply, the link stability and flawless communication depends directly over the energy of the devices. Routing protocols are responsible for ensuring energy efficient path discovery and try to reduce energy consumption of the nodes within the network. Major routing protocols minimize energy consumption by choosing minimum hop distance nodes, so as to enhance transmission rates or to reduce delay in transmissions [2]. Recent approaches in energy efficient routing concentrate on choosing specific nodes according to their offered residual energy, by which the protocol/ technique insured to attain energy efficiency with different limited network performance.

Researchers have found several improved solutions for achieving energy efficiency in these decentralized networks. A number of them provide routing with minimum energy utilization and aiding on lifetime maximization. Routing Protocols should uplift and retain network operations for longer time ensuring efficient ways between communicating nodes. Prolonged communication was achieved by minimizing node's energy consumption throughout its active and inactive states. Following are the ways used to achieve energy efficiency in mobile ad-hoc networks [3].

\section{FF-AOMDV AND DRAGONFLY TOPOLOGY}

\subsection{Fitness Function}

The fitness function is an improvement technique that comes as a part of the many optimization algorithmic rules like genetic algorithm, bee colony algorithmic rule, firefly algorithmic rule and particle swarm optimization rule. The fitness finds the most important factor of several factors 
necessary in the optimization method that counts on the aim of the analysis. In MANETs, the fitness factors are energy, distance, delay, bandwidth etc. This matches the reasons for designing any routing protocol, as they aim to enhance the full utilization of network resources. In this analysis, the fitness function used is Energy consumption in association with a type of Swarm Intelligence (SI) called Dragonfly Algorithm such as Particle Swarm optimization (PSO) rule. It had been used with wireless sensor networks to optimize the choice route in case the first route fails [1][16][17]. The factors that affect the selection of the optimum route are:

- The remaining energy functions for each node

- The distance functions of the links connecting the neighboring nodes

- Energy consumption of the nodes

- Communication delay of the nodes.

\subsection{FF-AOMDV}

In an old scenario of traditional AOMDV, once a RREQ is broadcasted by a source node, more than one route to the destination are found and the data packets are forwarded through these routes without knowing the routes' quality. By implementing the above explained rule on an analogous scenario, the routes selection is entirely different. Once a RREQ is broadcasted and received, the provision node will have three (3) forms of information to realize the selection of the shortest and optimized route with reduced energy consumption [1][17][18]. This information includes:

- Information about network's each node's energy level

- The distance of every route

- The energy consumed in the process of route discovery.

The route, that consumes less energy, may probably be (a) the route that has the shortest distance; (b) the route with the very best level of energy, or (c) both. The supply node can then send the information packets via the route with highest energy state, to minimize its energy consumption. Unlike of different multipath routing protocols, this protocol also initiates new route discovery method once all routes to the destination are unsuccessful. Within the event once the chosen route fails, the supply node can then select another route from its routing table that represents the shortest route with higher energy level and minimum energy consumption. The best route with less distance to destination can consume less energy.

\subsection{Draganfly Topology}

A novel swarm intelligence optimization technique called Dragonfly Algorithm (DA) is used.

In the traditional AOMDV, it builds multiple paths using RREQs. It does not take into account the energy for choosing the paths. Here the proposed protocol not only considers residual energy but also transmission power of nodes in paths selection to maximize the lifetime of networks. The proposed system consists of three stages:

\section{- Calculate residual energy}

- Calculate energy consumption in route discovery

- $\quad$ Find shortest route with higher residual energy
This algorithm is conscientious for deployment of nodes in an exacting area. This will position the nodes in the known area. [1] [7] [18] a case of Dragonfly topology based node deployment algorithm can be as follows:-

Dragonfly topology based node development has 4 routers (a), 2 number of terminals connected to each other (p) and 2 number of channels within which is use to join the other groups (h). In dragonfly topology no. of network terminals is known as:

$$
\mathrm{N}=\mathrm{a} * \mathrm{p}(\mathrm{ah}+1)
$$

that scales to $\mathrm{N}=144$.

To steadiness channel load on load-balanced traffic, the network should have $a=2 p=2 h$. Each of the router topology is based on randomized placement of nodes using node deployment algorithm.

The following symbols are used in our description of the dragonfly topology.

$$
\begin{aligned}
& N=\text { number of network terminal } \\
& p=\text { Number of terminals connected to each router } \\
& a=\text { Number of routers in each group } \\
& h=\text { Number of channels within each router used to connect } \\
& \text { to other groups }
\end{aligned}
$$

Within the event on route selection once the chosen route fails, the supply node can then select another route from its routing table that represents the shortest route with higher energy level and minimum energy consumption.

\section{PROPOSED METHODOLOGY}

This research implements a new multipath routing protocol called the FF-AOMDV routing protocol with Dragonfly topology, which is a combination of Fitness Function and the AOMDV protocol and dragonfly topology. In a normal scenario, when a RREQ is broadcasted by a source node, more than one route to the destination will be found and the data packets will be forwarded through these routes without knowing the routes' quality. By implementing the said algorithm on the same scenario, the route selection will be totally different. When a RREQ is broadcasted and received, the source node will have three (3) types of information in order to find the shortest and optimized route with minimized energy consumption.

In the traditional AOMDV, it builds multiple paths using RREQs. It does not take into account the energy for choosing the paths. Here the proposed protocol not only considers residual energy but also transmission power of nodes in paths selection to maximize the lifetime of networks. 


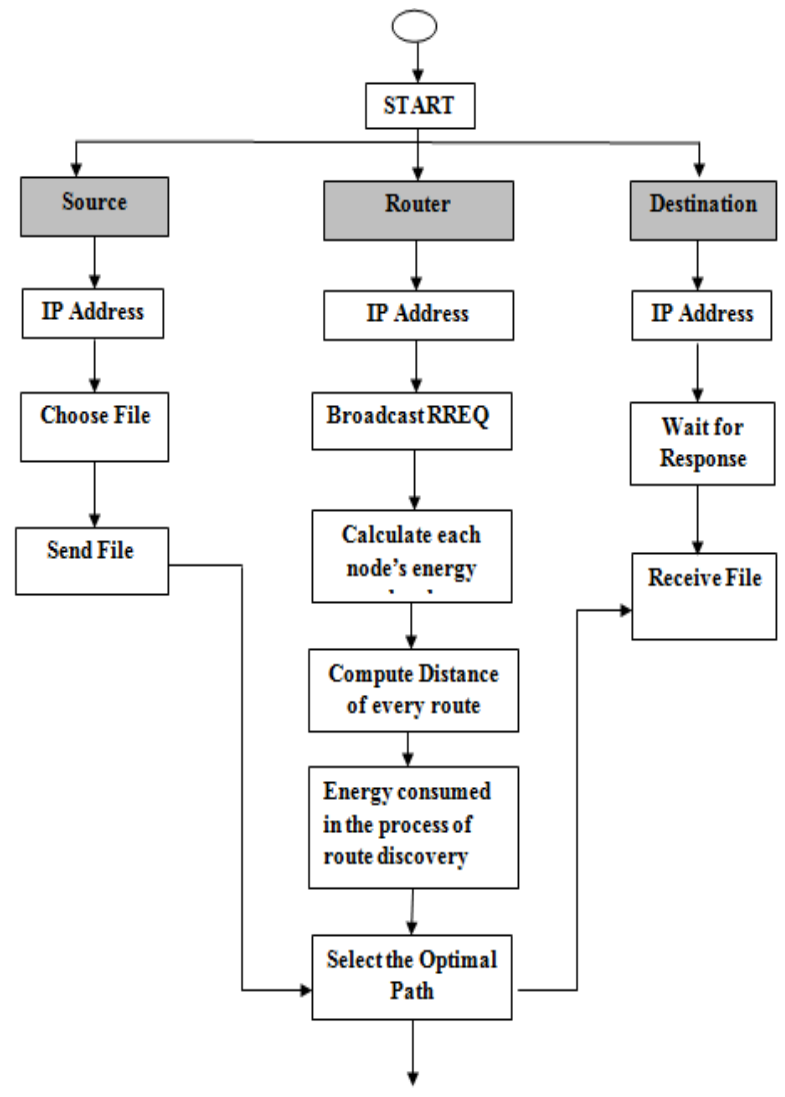

Fig.2 Flow diagram of proposed work

\section{SIMULATION RESULTS}

\subsection{Simulation Scenarios}

In this simulation model, we utilized the Constant Bit Rate (CBR) as a traffic source with 50 mobile nodes that are distributed randomly in a $860 \mathrm{~m} * 660 \mathrm{~m}$ network area; the network topology may therefore, undergo random change since the nodes' distribution and their movement are random. The transmission range of the nodes was set to $250 \mathrm{~m}$, while, for each node, the initial energy level was set to 100 joules. Three different scenarios were chosen to see how they are affecting the performance of the proposed FF-AOMDV protocol with Dragonfly topology. In the first scenario, we varied the packet size as $64,128,256,512,1024$ bytes and kept both the node speed and simulation time fixed as 5 meter/second and for 50 seconds respectively. All other network parameters are the same for all runs and for all simulated protocols. In the second scenario, we varied the node speed as $5,7.5,10,12.5,15$ seconds and kept the packet size and simulation time fixed as 256 bytes and 15 seconds respectively. Finally, in the third scenario, we varied the simulation time as 50,100, 150, 200, 250 seconds and kept the both the node speed and packet size fixed as 5 meters/second and 256 bytes respectively.

Table 1 Table of Various Simulation Scenarios

\begin{tabular}{|l|l|l|l|}
\hline S. No. & Scenario Elements & Values & Unit \\
\hline 1. & Number of runs & 5 & \\
\hline 2. & Number of nodes & 50 & Nodes \\
\hline
\end{tabular}

\begin{tabular}{|c|c|c|c|}
\hline 3. & Node speed & $\begin{array}{l}5,7.5,10 \\
12.5,15\end{array}$ & Meter/second \\
\hline 4. & Queue size & 50 & packets \\
\hline 5. & Simulation area & $860 * 660$ & Meter $^{2}$ \\
\hline 6. & Routing protocols & $\begin{array}{l}\text { FF- } \\
\text { AOMDV, } \\
\text { AOMR-LM, } \\
\text { AOMDV }\end{array}$ & Protocol \\
\hline 7. & Mobility model & $\begin{array}{l}\text { Random } \\
\text { way point }\end{array}$ & \\
\hline 8. & Packet size & $\begin{array}{l}64,128, \\
256,512 \\
1024\end{array}$ & Bytes \\
\hline 9. & Transmission range & 250 & Meter \\
\hline 10. & Traffic type & CBR & \\
\hline 11. & Initial energy & 100 & Joules \\
\hline 12. & $\begin{array}{l}\text { Transmission } \\
\text { power consumption }\end{array}$ & 0.02 & Joules \\
\hline 13. & $\begin{array}{l}\text { Receive power } \\
\text { consumption }\end{array}$ & 0.01 & Joules \\
\hline 14. & Sleep power & 0.001 & Joules \\
\hline 15. & Simulation time & $\begin{array}{l}50,100, \\
150,200, \\
250\end{array}$ & seconds \\
\hline
\end{tabular}

\subsection{Performance Parameters}

To evaluate the performance of our proposed algorithm, three different scenarios were selected (i.e. node speed, packet size, and simulation time) as explained above. The performance metrics considered here are:

\subsubsection{Packet Delivery Ratio (PDR) $\frac{\text { numberof packets received }}{\text { numberof packetssent }} \times 100_{\mathrm{It}}$ is the ratio of} the data packets that were delivered to the destination node to the data packets that were generated by the source. The higher the ratio, the better the performance of the routing protocol.

$\mathrm{PDR}=$

Fig. 3 shows graph of packet delivery ratio with simulation time. The fig. shows the variation of packet delivery ratio on varying simulation time for FF-AOMDV with Dragonfly topology, AOMR-LM and AOMDV routing protocols. Simulation time is varied as 50,100, 150, 200 and 250 seconds.

When the simulation time increases, the packet delivery ratio also increases. The FF-AOMDV with Dragonfly topology has better performance in terms of packet delivery ratio than both AOMR-LM and AOMDV protocols. The FF-AOMDV protocol with Dragonfly topology achieved $76 \%$ of packet delivery ratio in 50 seconds of simulation time and $97 \%$ in 250 second of simulation, the AOMR-LM protocol achieved 
$70.23 \%$ of packet delivery ratio in 50 seconds simulation time and $76.2 \%$ in 250 seconds of simulation time and finally, the AOMDV achieved $74.8 \%$ in 50 seconds simulation time and $78.7 \%$ of 250 seconds simulation time. The FF-AOMDV with Dragonfly topology has higher PDR due to having multiple paths always available in case of any chance or case of route failure.

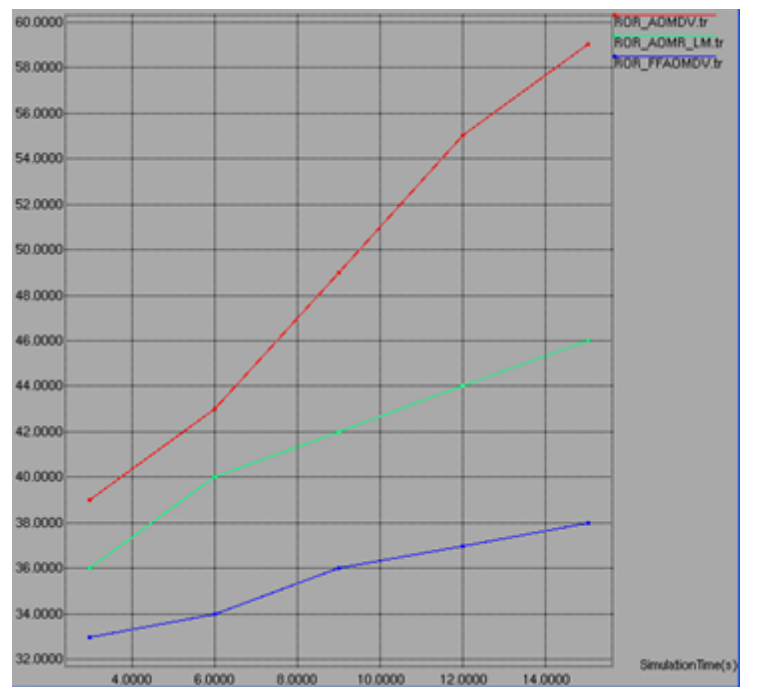

Fig.3 Graph of packet delivery ratio with simulation time

\subsubsection{Throughput}

Throughput is known as the number of bits that the destination has successfully received.

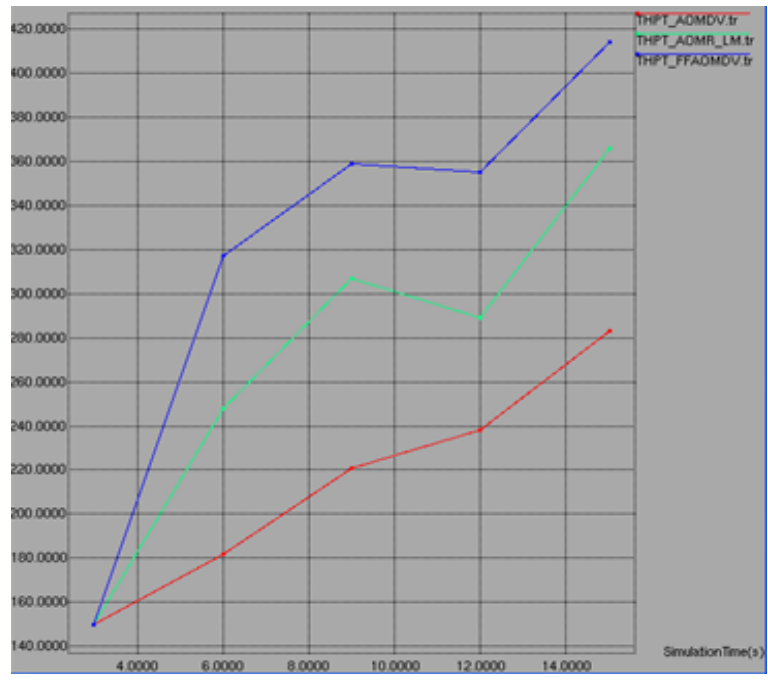

Fig.4 Graph of throughput

Fig.4 shows the comparison of throughput behalf of simulation time. In this figure $\mathrm{x}$ axis show the simulation time and $y$ axis show the throughput. In this fig. shows the effect on the throughput on varying simulation time for FF-AOMDV with Dragonfly topology, AOMR-LM and AOMDV routing protocols. Simulation time is varied as 50, 100, 150, 200 and 250 seconds. When the simulation time increases, the throughput also increases. The FF-AOMDV protocol with Dragonfly topology has better performance in terms of throughput than both AOMR-LM and AOMDV protocols. The FF-AOMDV with Dragonfly topology has $171 \mathrm{kbps}$ throughput in 50 second simulation time and $1120 \mathrm{kbps}$ in 250 second of simulation time, the AOMR-LM has $126.67 \mathrm{kbps}$ throughput in 50 second simulation time and $1090 \mathrm{kbps}$ in 250 second simulation time and finally, the AOMDV has 104.78 kbps throughput in 50 second simulation time and $902 \mathrm{kbps}$ in 250 second simulation time.

In FF-AOMDV with Dragonfly topology the packet-loss is nearly zero because of its unique property of storing the information about the various energy efficient paths available for flawless communication.

\subsubsection{End-to-End delay}

End-to-End delay refers to the average time taken by data packets in successfully transmitting messages across the network from source to destination. This includes all types of delays, such as packet queuing at interface queue; propagation time and transfer time; and buffering during the route discovery latency.

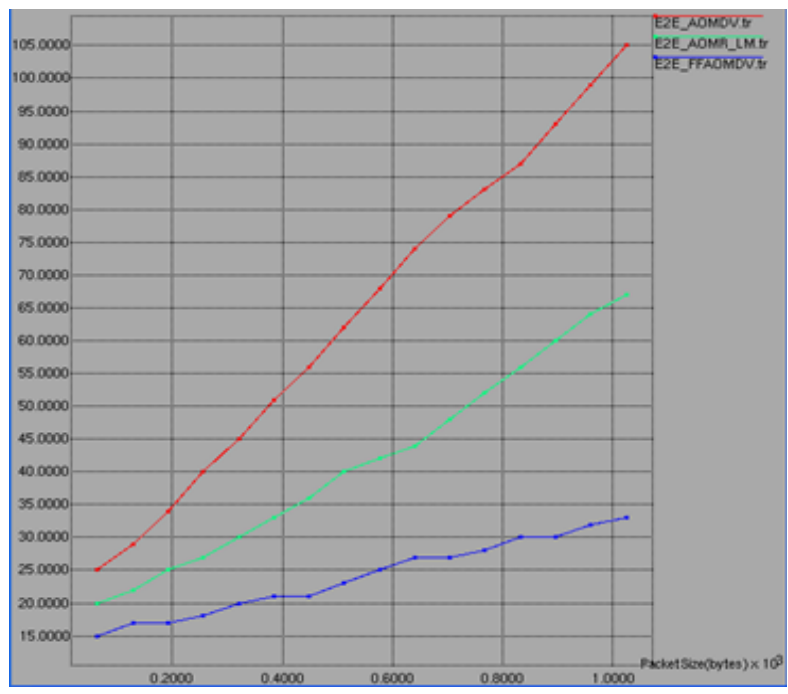

Fig.5 Graph of End to end delay

Fig.5 shows the comparison of E2E delay w.r.t. packet size. The fig. shows the change of end-to-end delay for FFAOMDV with Dragonfly topology, AOMR-LM and AOMDV. When the packet size increases as 64, 128, 256, 512,1024 bytes, the end-to-end delay also increases. The E2E delay in FF-AOMDV routing protocol with Dragonfly topology increases from $15 \mathrm{~ms}$ to $26 \mathrm{~ms}$, in the AOMR-LM protocol it increases from $18.64 \mathrm{~ms}$ to $44 \mathrm{~ms}$ and finally, in the AOMDV protocol it increases from $21.63 \mathrm{~ms}$ to $42 \mathrm{~ms}$. The FF-AOMDV routing protocol with Dragonfly topology has better performance than both AOMR-LM and AOMDV in terms of end-to-end delay. In FF-AOMDV with Dragonfly topology the E2E delay is minimum because it has the most energy efficient multiple paths to avoid delay in transmission by reducing the packet queue size and transmitting packets through multiple routes.

\subsubsection{Energy Consumption}

Energy consumption refers to the amount of energy that is spent by the network nodes within the simulation time. This is obtained by calculating each node's energy level at the end of the simulation, factoring in the initial energy of each one.

Fig.6 shows the comparison graph of energy consumption behalf of node speed. The variation in energy consumption for FF-AOMDV with Dragonfly topology, AOMR-LM and AOMDV are shown. When the node speed increases as 5, 7.5, 
$10,12.5,15 \mathrm{~m} / \mathrm{s}$, the energy consumption also increases. In the FF-AOMDV with Dragonfly topology it increases from 60 joules to 98 joules as it is designed to select the path having higher energy levels and shortest route from source to destination, in AOMR-LM it increases from 61 joules to 112 joules and in AOMDV it increases from 72 joules to 158 joules.

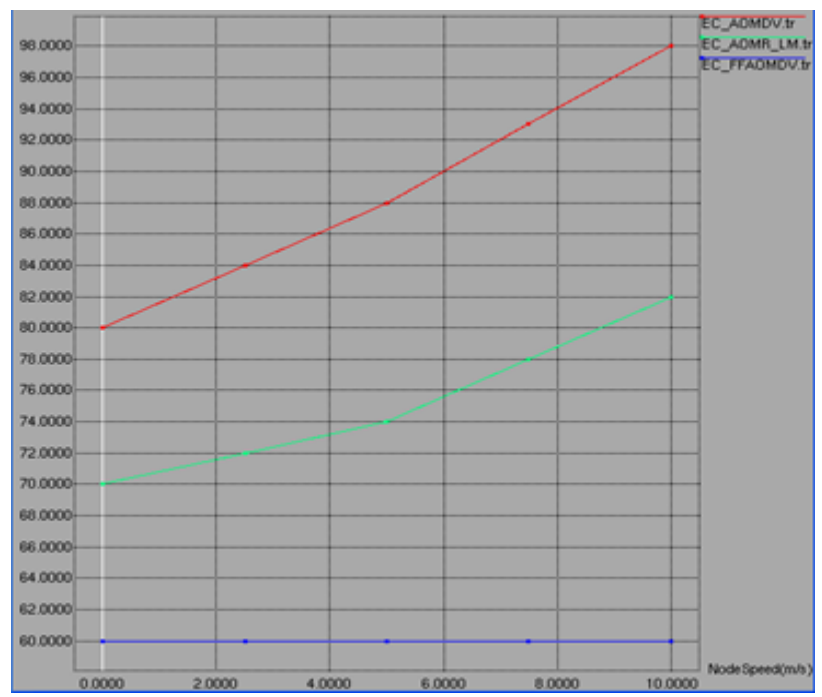

Fig.6 Graph of energy consumption behalf of node speed

The FF-AOMDV with Dragonfly topology has least energy consumption because it has the information of most energy efficient paths stored. It sends the data packets through the route with highest energy and minimum source to destination distance. Also it provides the facility of route transition in case of any route failure.

\subsubsection{Network Lifetime}

The network lifetime refers to the required time for exhausting the battery of $\mathrm{n}$ mobile nodes.

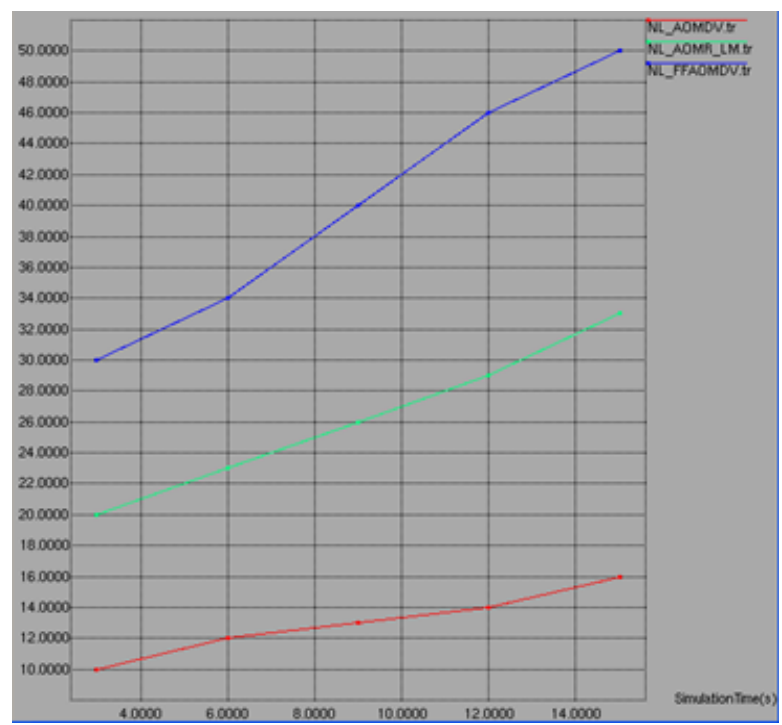

Fig.7 Graph of network lifetime

Fig.7 shows the comparison of network lifetime behalf of simulation time. In this figure $\mathrm{x}$ axis show the simulation time and $y$ axis shows the number of exhausted nodes for FFAOMDV with Dragonfly topology, AOMR-LM and AOMDV when varying the simulation time. The FF-AOMDV with Dragonfly topology exhausts 0 nodes in 50 seconds and 2 nodes in 250 seconds, the AOMR-LM exhausts 0 nodes in 50 seconds and 3 nodes in 250 seconds, while, the AOMDV exhausts 2 nodes in 50 seconds but 6 nodes in 250 seconds. The FF-AOMDV with Dragonfly topology enhances its network lifetime as it routes the traffic to the nodes having higher energy in the network. In the case, when the energy of these nodes get exhausted the topology has the property of storing information about various energy efficient routes and hence it transfers the traffic to next energy efficient shortest path, thus enhancing the network lifetime.

\subsubsection{Routing Overhead Ratio}

The routing overhead ratio metric is the total number of routing packets, which is divided by the overall number of data packets that were delivered. The routing overhead has an effect on the network's robustness in terms of the bandwidth utilization and battery power consumption of the nodes.

Fig.8 shows the comparison of routing overhead ratio w.r.t. simulation time. The graph shows a clear improvement in routing overhead ratio in FF-AOMDV with Dragonfly topology as compared to AOMR-LM and AOMDV since it is showing minimum Routing overhead when compared to the existing protocols.

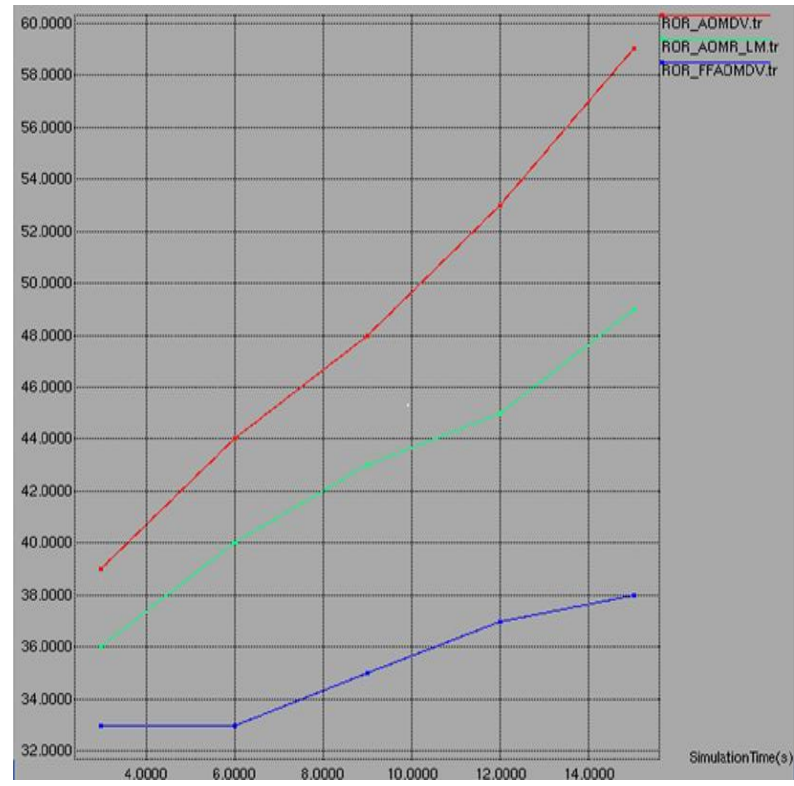

Fig.8 Graph of routing overhead ratio

The FF-AOMDV with Dragonfly topology has improved Routing overhead ratio as it provides multiple paths always ready for the data packets to travel through in case of any route failure and hence reducing the overhead due to control packets and queuing of data packets, thus improving Routing overhead ratio.

Now that we have seen all the improvements in performance parameters in graphical form, we can easily take the data values from the graphs and can compare them in tabular form. The following are the tables in which we have compared the performance metrics on varying simulation scenarios. All the tables show the comparison of various existing protocols (single run) with the proposed FF-AOMDV with Dragonfly topology (three runs) over each value of varying simulation values to show the justified values of the proposed algorithm. 
Table 2 Comparison of Energy Consumption

\begin{tabular}{|c|c|c|c|}
\hline & $\begin{array}{c}\text { Existing } \\
\text { Algorithms }\end{array}$ & \multirow[t]{2}{*}{$\begin{array}{l}\text { Node Speed } \\
(\mathrm{m} / \mathrm{s})\end{array}$} & $\begin{array}{l}\text { Proposed } \\
\text { Algorithm }\end{array}$ \\
\hline & $\begin{array}{c}\text { Energy } \\
\text { Consumption } \\
\text { (joules) }\end{array}$ & & $\begin{array}{c}\text { Energy } \\
\text { Consumption } \\
\text { (joules) }\end{array}$ \\
\hline FFAOMDV & 63 & \multirow{3}{*}{5} & 60 \\
\hline AOMDV & 72 & & 61 \\
\hline AOMR-LM & 61 & & 60 \\
\hline FFAOMDV & 74 & \multirow{3}{*}{7.5} & 65 \\
\hline AOMDV & 82 & & 64 \\
\hline AOMR-LM & 68 & & 66 \\
\hline FFAOMDV & 80 & \multirow{3}{*}{10} & 75 \\
\hline AOMDV & 92 & & 74 \\
\hline AOMR-LM & 79 & & 76 \\
\hline FFAOMDV & 90 & \multirow{3}{*}{12.5} & 79 \\
\hline AOMDV & 122 & & 81 \\
\hline AOMR-LM & 89 & & 80 \\
\hline FFAOMDV & 120 & \multirow{3}{*}{15} & 96 \\
\hline AOMDV & 158 & & 95 \\
\hline AOMR-LM & 112 & & 98 \\
\hline
\end{tabular}

Table 3 Comparison of PDR

\begin{tabular}{|c|c|c|c|}
\hline & $\begin{array}{c}\text { Existing } \\
\text { Algorithms }\end{array}$ & \multirow{2}{*}{$\begin{array}{c}\text { Simulation } \\
\text { time } \\
\text { (seconds })\end{array}$} & $\begin{array}{c}\text { Proposed } \\
\text { Algorithm }\end{array}$ \\
& PDR $\%$ & & PDR $\%$ \\
\hline FFAOMDV & $75.36 \%$ & \multirow{4}{*}{50} & $76 \%$ \\
\hline AOMDV & $74.8 \%$ & & $76.5 \%$ \\
\hline AOMR-LM & $70.23 \%$ & & $75.81 \%$ \\
\hline FFAOMDV & $76.8 \%$ & \multirow{4}{*}{100} & $82 \%$ \\
\hline AOMDV & $75 \%$ & & $80 \%$ \\
\hline AOMR-LM & $70.8 \%$ & & $81 \%$ \\
\hline FFAOMDV & $76.8 \%$ & \multirow{4}{*}{150} & $88 \%$ \\
\hline AOMDV & $76.2 \%$ & & $84 \%$ \\
\hline AOMR-LM & $72 \%$ & & $86 \%$ \\
\hline FFAOMDV & $77.1 \%$ & \multirow{4}{*}{200} & $88 \%$ \\
\hline AOMDV & $76.8 \%$ & & $85 \%$ \\
\hline AOMR-LM & $76 \%$ & & $84 \%$ \\
\hline FFAOMDV & $78 \%$ & \multirow{2}{*}{250} & $100 \%$ \\
\hline AOMDV & $77.4 \%$ & & $96 \%$ \\
\hline AOMR-LM & $76.2 \%$ & & $97 \%$ \\
\hline
\end{tabular}

Table 4 Comparison of Network lifetime

\begin{tabular}{|c|c|c|c|}
\hline & $\begin{array}{c}\text { Existing } \\
\text { Algorithms }\end{array}$ & \multirow{2}{*}{$\begin{array}{l}\text { Simulation } \\
\text { time } \\
\text { (seconds) }\end{array}$} & $\begin{array}{l}\text { Proposed } \\
\text { Algorithm }\end{array}$ \\
\hline & $\begin{array}{c}\text { Exhausted } \\
\text { Nodes }\end{array}$ & & $\begin{array}{c}\text { Exhausted } \\
\text { Nodes }\end{array}$ \\
\hline FFAOMDV & 0 & \multirow{3}{*}{50} & 0 \\
\hline AOMDV & 2 & & 0 \\
\hline AOMR-LM & 0 & & 0 \\
\hline FFAOMDV & 0 & \multirow{3}{*}{100} & 0 \\
\hline AOMDV & 3 & & 0 \\
\hline AOMR-LM & 0 & & 0 \\
\hline FFAOMDV & 1 & \multirow{3}{*}{150} & 0 \\
\hline AOMDV & 2 & & 1 \\
\hline AOMR-LM & 0 & & 0 \\
\hline FFAOMDV & 2 & \multirow{3}{*}{200} & 1 \\
\hline AOMDV & 4 & & 1 \\
\hline AOMR-LM & 1 & & 1 \\
\hline FFAOMDV & 3 & \multirow{3}{*}{250} & 2 \\
\hline AOMDV & 6 & & 2 \\
\hline AOMR-LM & 2 & & 1 \\
\hline
\end{tabular}

Table 5 Comparison of Throughput

\begin{tabular}{|c|c|c|c|}
\hline & $\begin{array}{c}\text { Existing } \\
\text { Algorithms }\end{array}$ & \multirow{2}{*}{$\begin{array}{c}\text { Simulation } \\
\text { time } \\
\text { (seconds) }\end{array}$} & $\begin{array}{c}\text { Proposed } \\
\text { Algorithm }\end{array}$ \\
\cline { 2 - 2 } & $\begin{array}{c}\text { Throughput } \\
\text { (Kbps) }\end{array}$ & & $\begin{array}{c}\text { Throughput } \\
\text { (Kbps) }\end{array}$ \\
& & & \\
\hline FFAOMDV & 140.78 & \multirow{2}{*}{50} & 171 \\
\hline AOMDV & 104.78 & & 160 \\
\hline AOMR-LM & 126.67 & & 182.52 \\
\hline FFAOMDV & 367.68 & \multirow{2}{*}{100} & 380 \\
\hline AOMDV & 300 & & 392 \\
\hline AOMR-LM & 360 & & 382.67 \\
\hline FFAOMDV & 400 & \multirow{2}{*}{150} & 440 \\
\hline AOMDV & 390 & & 410 \\
\hline AOMR-LM & 398 & \multirow{2}{*}{200} & 448 \\
\hline FFAOMDV & 996 & & 1108 \\
\hline AOMDV & 740 & & 1028 \\
\hline AOMR-LM & 856 & \multirow{2}{*}{250} & 1044 \\
\hline FFAOMDV & 1100 & & 1175 \\
\hline AOMDV & 902 & & 1029 \\
\hline AOMR-LM & 1090 & & 1120 \\
\hline
\end{tabular}

Table 6 Comparison of E2E Delay

\begin{tabular}{|c|c|c|c|}
\hline & $\begin{array}{c}\text { Existing } \\
\text { Algorithms }\end{array}$ & \multirow[t]{2}{*}{$\begin{array}{c}\text { Packet size } \\
\text { (bytes) }\end{array}$} & $\begin{array}{l}\text { Proposed } \\
\text { Algorithm }\end{array}$ \\
\hline & $\begin{array}{l}\text { E2E delay } \\
(\mathrm{ms})\end{array}$ & & $\begin{array}{l}\text { E2E delay } \\
(\mathrm{ms})\end{array}$ \\
\hline FFAOMDV & 17.53 & \multirow[t]{3}{*}{64} & 15 \\
\hline AOMDV & 21.63 & & 16 \\
\hline AOMR-LM & 18.64 & & 15 \\
\hline FFAOMDV & 24 & \multirow[t]{3}{*}{128} & 17 \\
\hline AOMDV & 27 & & 17 \\
\hline AOMR-LM & 25 & & 16 \\
\hline FFAOMDV & 26 & \multirow[t]{3}{*}{256} & 19 \\
\hline AOMDV & 29 & & 20 \\
\hline AOMR-LM & 27 & & 21 \\
\hline FFAOMDV & 27 & \multirow[t]{3}{*}{512} & 20 \\
\hline AOMDV & 32 & & 22 \\
\hline AOMR-LM & 28 & & 22 \\
\hline FFAOMDV & 32 & \multirow[t]{3}{*}{1024} & 23 \\
\hline AOMDV & 42 & & 24 \\
\hline AOMR-LM & 44 & & 26 \\
\hline
\end{tabular}

Table 7 Comparison of ROR

\begin{tabular}{|c|c|c|c|}
\hline & $\begin{array}{c}\text { Existing } \\
\text { Algorithms }\end{array}$ & \multirow{2}{*}{$\begin{array}{c}\text { Simulation } \\
\text { time } \\
\text { (seconds) }\end{array}$} & $\begin{array}{c}\text { Proposed } \\
\text { Algorithm }\end{array}$ \\
\cline { 2 - 2 } & ROR (\%) & & ROR $\%$ \\
\hline FFAOMDV & 33 & \multirow{2}{*}{50} & 31.97 \\
\hline AOMDV & 40.89 & & 35.74 \\
\hline AOMR-LM & 37.65 & & 34.19 \\
\hline FFAOMDV & 33 & \multirow{2}{*}{100} & 32.43 \\
\hline AOMDV & 44 & & 37.38 \\
\hline AOMR-LM & 40 & & 35.57 \\
\hline FFAOMDV & 44.72 & \multirow{2}{*}{150} & 38 \\
\hline AOMDV & 46.8 & & 39 \\
\hline AOMR-LM & 42 & & 39.94 \\
\hline FFAOMDV & 45.62 & \multirow{2}{*}{200} & 41.72 \\
\hline AOMDV & 49.76 & & 43.42 \\
\hline AOMR-LM & 43.68 & & 42.23 \\
\hline FFAOMDV & 43 & \multirow{2}{*}{250} & 44.15 \\
\hline AOMDV & 53 & & 47.21 \\
\hline AOMR-LM & 48 & & 45.57 \\
\hline
\end{tabular}




\section{CONCLUSION}

In this research, we proposed a new energy efficient multipath routing algorithm called FF-AOMDV with Dragonfly topology simulated using NS-2 under three different scenarios, varying node speed, and packet size and simulation time. These scenarios were tested by six performance metrics Packet delivery ratio, Throughput, End-to-end-delay, Routing overhead ratio, Energy consumption and Network lifetime. Simulation results showed that the proposed FF-AOMDV with Dragonfly topology has performed better than the existing FF-AOMDV and the other two protocols AOMR-LM and AOMDV in throughput, packet delivery ratio, routing overhead ratio and end-to-end delay. It also performed well against FF-AOMDV for conserving more energy and enhancing the network lifetime.

\section{REFERENCES}

[1] Mueen Uddin, Aqeel Taha, Raed Alsaqour and Tanzila Saba , Energy Efficient Multipath Routing Protocol for Mobile ad-hoc Network Using the Fitness Function, IEEE ACCESS, Volume 5, May 2017

[2] J. Sharma, Dhirendra Kumar, Amar Nath Patra, and Chiranjeev Kumar. "An update based energy-efficient reactive routing protocol for mobile Ad Hoc networks." International Journal of Computer Network and Information Security 5.11 (2013): 17.

[3] Tanaka, Smail Omar, et al., A multipath energyconserving routing protocol for wireless ad hoc networks lifetime improvement, I.J. Intelligent Systems and Applications, 2016, 06, 61-69 Published Online May 2016 in MECS DOI: 10.5815/ijisa.2016.06.10

[4] Hassanali Nasehi et al., Improving Energy Efficiency In MANETs By Multi-path Routing, International Journal of Wireless \& Mobile Networks (IJWMN) Vol. 5, No. 1, February 2013

[5] Chaba, Yogesh, R. B. Patel, and Rajesh Gargi. "Issues and challenges involved in multipath routing with DYMO protocol." International Journal of Information Technology and Knowledge Management 5.1 (2012): 21-25.

[6] Md Azharuddin et al., PSO-based approach for energyefficient and energy-balanced routing and clustering in wireless sensor networks, Methodologies and Application soft computing, Vol.21 Issue 22/2017

[7] Muamer N. Mohammed, Nassir S. Kadhim and Waleed Kh. Ahmed, An Energy Efficient Multipath Routing Protocol Based On Signal Strength For Mobile Ad-hoc Network, ARPN Journal of Engineering and Applied Sciences, VOL. 11, NO. 11, JUNE 2016 ISSN 18196608

[8] Taha, Aqeel, et al. "Energy efficient multipath routing protocol for mobile ad-hoc network using the fitness function." IEEE Access 5 (2017): 10369-10381.

[9] Azharuddin, Md, and Prasanta K. Jana. "PSO-based approach for energy-efficient and energy-balanced routing and clustering in wireless sensor networks." Soft Computing 21.22 (2017): 6825-6839.

[10] Devi, V. Sowmya, and Nagaratna P. Hegde. "Energy efficient multipath routing protocol for enhancing QoS and QoE in multimedia applications for MANETs."
International Journal of Communication Networks and Information Security 8.3 (2016): 158.

[11] C.Hema, Sharmila Sankar and Sandhya, Energy Efficient Cluster based Protocol to Extend the RFID Network Lifetime using Dragonfly Algorithm, International Conference on Communication and Signal Processing, April 6-8, 2016

[12] Jayavenkatesan, Rangaraj, and Anitha Mariappan. "ENERGY EFFICIENT MULTIPATH ROUTING FOR MANET BASED ON HYBRID ACO-FDRPSO."

[13] Ashrafuddin, Md, Md Manowarul Islam, and $\mathrm{Md}$ Mamun-or-Rashid. "Energy efficient fitness based routing protocol for underwater sensor network." International Journal of Intelligent Systems and Applications 5.6 (2013): 61.

[14] Jahanbakhsh Gudakahriz, Sajjad, Shahram Jamali, and Mina Vajed Khiavi. "Energy efficient routing in mobile ad hoc networks by using honey bee mating optimization." Journal of advances in computer research 3.4 (2012): 77-87.

[15] Sajjad Jahanbakhsh Gudakahriz et al., Energy Efficient Routing in Mobile Ad Hoc Networks by Using Honey Bee Mating Optimization, Journal of Applied Research and Technology ISSN: 1665-6423 vol. 11, N0. 6, December 2013

[16] Chen, H. H., Li, G. Q., \& Liao, H. L. (2009, August). A self-adaptive improved particle swarm optimization algorithm and its application in available transfer capability calculation. In 2009 Fifth International Conference on Natural Computation (Vol. 3, pp. 200205). IEEE

[17] Trelea, I. C. 2003. The particle swarm optimization algorithm: convergence analysis and parameter selection. Information processing letters 85(6): 317-325.

[18] Hiremath, P. S., \& Joshi, S. M. (2012). Energy efficient routing protocol with adaptive fuzzy threshold energy for MANETs. International Journal of Computer Networks and Wireless Communications (IJCNWC), ISSN: 2250$3501 \mathrm{Vol}, 2$.

[19] De Rango, F., Guerriero, F., \& Fazio, P. (2012). Linkstability and energy aware routing protocol in distributed wireless networks. Parallel and Distributed Systems, IEEE Transactions on, 23(4), 713-726.

[20] Manickavelu, D., \& Vaidyanathan, R. U. (2014). Particle swarm optimization (PSO)-based node and link lifetime prediction algorithm for route recovery in MANET. EURASIP Journal on Wireless Communications and Networking, 2014 (1), 1-10.

[21] Mueller, S., Tsang, R. P., \& Ghosal, D. (2004). Multipath routing in mobile ad hoc networks: Issues and challenges. In Performance tools and applications to networked systems (pp. 209-234). Springer Berlin Heidelberg.

[22] Balaji, V., \& Duraisamy, V. (2011). Varying Overhead Ad Hoc on Demand Vector Routing in Highly Mobile Ad Hoc Network. Journal of Computer Science, 7(5), pp. 678-682 
[23] Giordano, S. (2002). Mobile ad hoc networks. Handbook of wireless networks and mobile computing, 325-346.

[24] Zheng, S., Weiqiang, W. U., \& Zhang, Q. (2011). Energy and link-state based routing protocol for MANET. IEICE TRANSACTIONS on Information and Systems, 94(5), 1026-1034.
[25] Marina, M. K., \& Das, S. R. (2006). Ad hoc on-demand multipath distance vector routing. Wireless communications and mobile computing, 6(7), 956 Spector, A. Z. 1989. Achieving application requirements. In Distributed Systems, S. Mullender. 